\title{
Ultrahigh Durability Perovskite Solar Cells
}

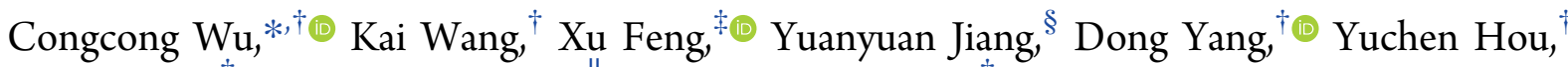 \\ Yongke Yan, ${ }^{\dagger}$ Mohan Sanghadasa, ${ }^{\|}$and Shashank Priya ${ }^{*}, \dagger$ \\ ${ }^{\dagger}$ Materials Science and Engineering, Pennsylvania State University, University Park, Pennsylvania 16802, United States \\ ${ }^{\ddagger}$ Surface Analysis Laboratory, Department of Chemistry and ${ }^{\S}$ Center for Energy Harvesting Materials and System (CEHMS), \\ Virginia Polytechnic Institute and State University, Blacksburg, Virginia 24061, United States \\ "Aviation and Missile Research, Development, and Engineering Center, United States Army RDECOM, Redstone Arsenal, Alabama \\ 35898, United States
}

\section{Supporting Information}

ABSTRACT: Unprecedented conversion efficiency has been demonstrated for perovskite solar cells (PSCs), however, their stability and reliability continue to be challenge. Here, an effective and practical method is demonstrated to overcome the device stability issues in PSCs. $\mathrm{A} \mathrm{CF}_{4}$ plasma treatment method is developed that results in the formation of a robust $\mathrm{C}-\mathrm{F}_{x}$ layer covering the PSC device, thereby, imparting protection during the operation of solar cell. PSCs exposed to fluorination process showed excellent stability against water, light, and oxygen, displaying relatively no noticeable degradation after being dipped into water for considerable
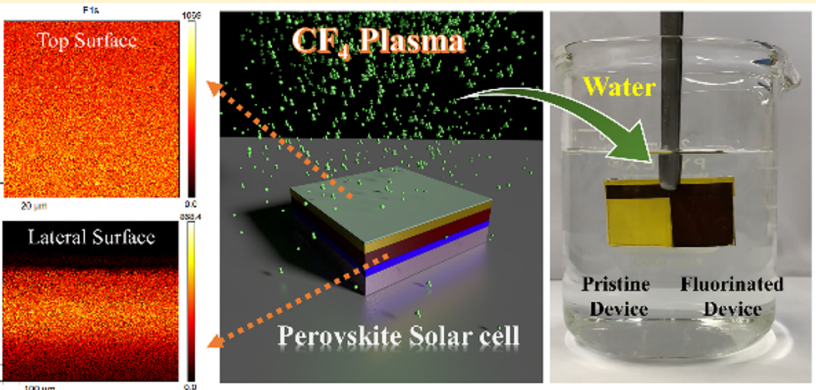
time period. The fluorination process did not have any impact on the morphology and electrical property of the top Spiro-OMeTAD layer, resulting in a conversion efficiency of $18.7 \%$, which is identical to that of the pristine PSC. Under the continuous Xe lamp (AM 1.5G, 1 sun) illumination in ambient air for $100 \mathrm{~h}$, the fluorinated PSCs demonstrated $70 \%$ of initial conversion efficiency, which is $4000 \%$ higher than that of the pristine PSC devices. We believe this breakthrough will have significant impact on the transition of PSCs into real world applications.

KEYWORDS: Plasma, fluorination, perovskite, hydrophobicity, protection, stability

Power conversion efficiency (PCE) and lifespan of solar cells are two important factors affecting the economics of photovoltaics. Lead halide perovskite solar cells (PSCs) are promising technology as they have shown unprecedented conversion efficiency improvement from $3 \%$ to $23.7 \%$ in less than a decade. ${ }^{1,2}$ However, the long-term stability of PSCs remains the critical challenge that is limiting their transition. The perovskite photoactive layer in PSCs has severe stability issue, as it is sensitive to various environmental stimuli such as moisture, oxygen, heat, and UV light, and so forth, potentially causing irreversible degradation of PSCs. ${ }^{3-6}$ To address the stability issue of perovskite layer, various processing approaches have been suggested such as physical encapsulation, ${ }^{7-9}$ composition optimization ${ }^{10-12}$ and interface modification. ${ }^{13-16}$ Through these approaches, the perovskite endurance in high humid air has been improved from several hours to several months. ${ }^{14,17}$ In addition to the perovskite light absorber, PSC contains electron and hole transport materials (ETM and HTM, respectively). These charge carrier transport layers may also trigger and accelerate the degradation of PSCs. For instance, most PSCs employ organic molecules such as $2,2^{\prime}, 7,7^{\prime}$-tetrakis $(N, N$-dipmethoxyphenyl-amine $) 9,9^{\prime}$-spirobifluorene (Sprio-OMeTAD) and poly(triarylamine) (PTAA) as the HTM. In order to achieve high electrical conductance, the bis(trifluoromethane)sulfonimide lithium salt (Li-TFSI) and 4tert-butylpyridine ( $\mathrm{t}-\mathrm{BP})$ are typically used as dopants in the state-of-the-art HTM. However, the ionic dopant Li-TFSI is a highly deliquescent salt and the doped HTM can easily adsorb water, which will accelerate the decomposition of the underneath perovskite layer by percolating water. ${ }^{18-20}$ In order to improve the stability of PSC devices, not only the perovskite light absorbing layer but also all the functional layers, interface, and boundary should be taken into consideration.

To enhance the stability of PSC devices, especially their moisture resistance, we demonstrate the fluorination method to treat the surface that imparts high hydrophobicity. Bella et al. have shown the influence of fluoropolymers on PSCs that resulted in stable performance for up to 6 months. ${ }^{21}$ Kim et al. demonstrated that edged-selectively fluorine-functionalized graphene based electron transport layer results in stability improvement. ${ }^{22}$ Luo et al. employed a fluorine-containing hydrophobic Lewis acid as the dopant in HTM and demonstrated improvement in stability. ${ }^{23}$ The results from

Received: November 29, 2018

Revised: January 20, 2019

Published: January 29, 2019 
(a)

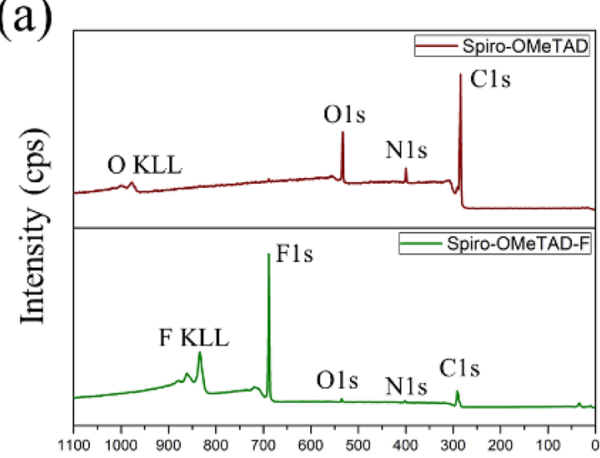

Binding Energy $(\mathrm{eV})$

(d)

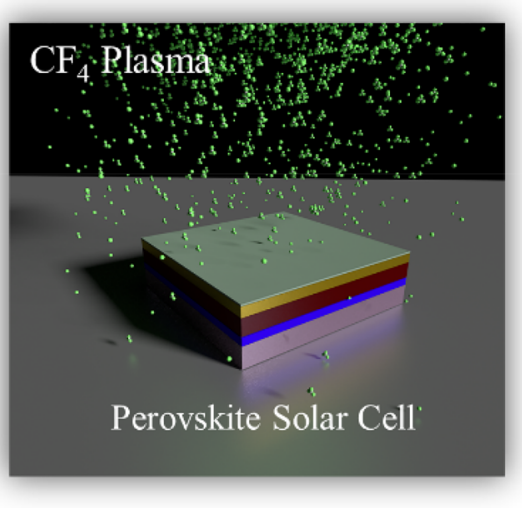

(b)

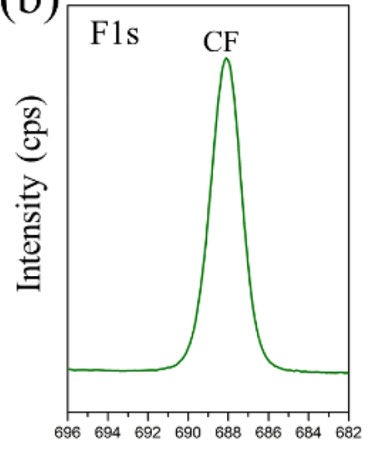

Binding Energy (eV) (c)

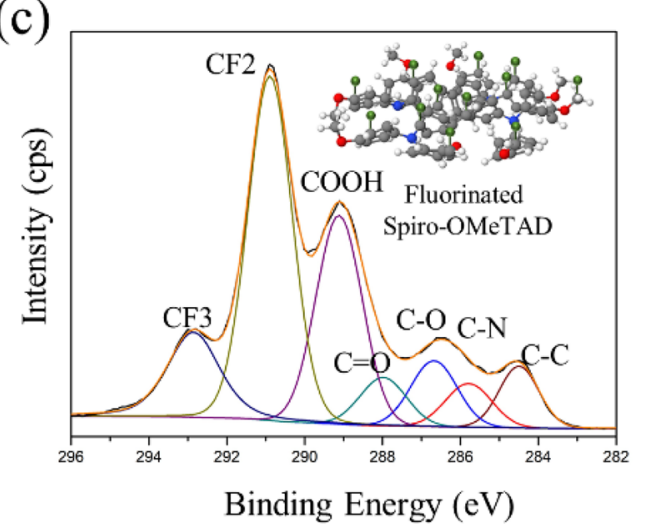

F1s

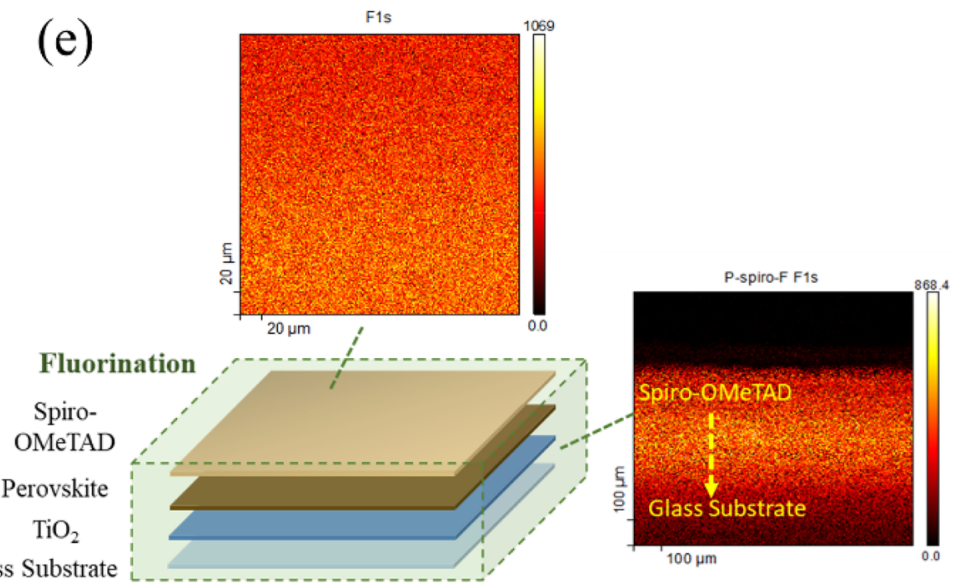

Glass Substrate

(g)
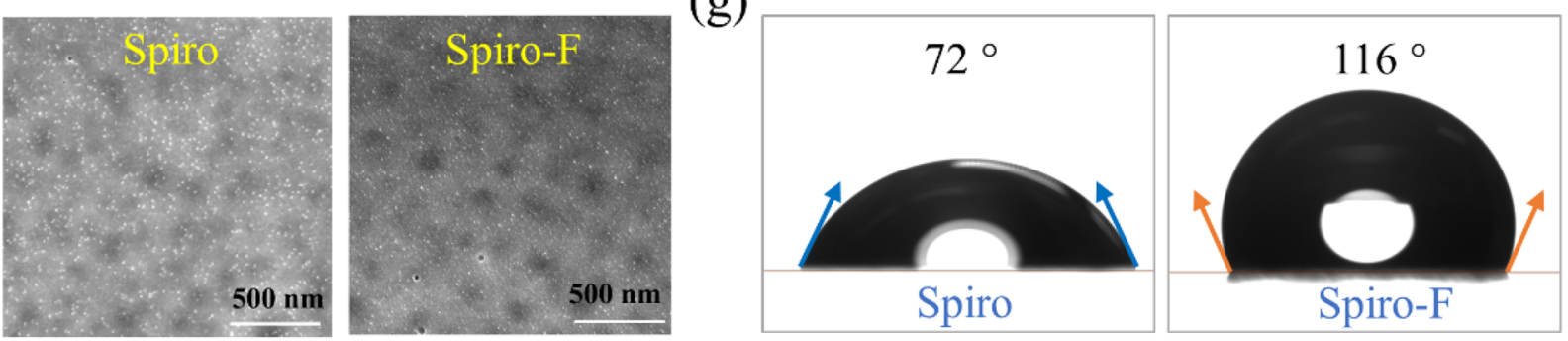

Figure 1. Chemical coordination between fluorine and PSCs with plasma fluorination treatment. (a) XPS survey scan of the Spiro-OMeTAD films with and without fluorination. High-resolution XPS spectrum of the fluorinated Spiro-OMeTAD film in binding energy region of (b) F 1s, 682$696 \mathrm{eV}$, and (c) C 1s, 280-300 eV. (d) Schematic illustration of the fluorination of PSC using CF 4 plasma. (e) F 1s XPS mapping of the top surface and lateral surface of PSCs device. (f) SEM and (g) water contact angle of Spiro-OMeTAD films with and without fluorination.

these prior studies indicate the effectiveness of fluorine incorporation but the level of improvement might vary depending upon the specific method used for fluorine incorporation within the device. In prior studies, the fluorine complex was either used as the capping layer or as additive in the charge transport layer, which could only improve the moisture resistance of one component or isolate the ambient moisture from the device in one dimension in the out-of-plane direction. Wang et al. demonstrated that lateral diffusion and in-plane corrosion of the perovskite layer can also induce fast device degradation. ${ }^{24}$ Thus, not only the top surface but also the lateral surfaces should be nanoencapsulated by fluorination treatment to prevent the moisture invasion so as to achieve a full protection for PSC devices.

Here, we provide a facile and effective approach for fluorinating the whole PSC device, which can provide complete protection against environmental degradation. The
$\mathrm{CF}_{4}$ plasma is used for fluorination treatment, where the radical fluorine $(\mathrm{F})$ atoms form durable $\mathrm{C}-\mathrm{F}$ bonds with the $\mathrm{C}$ atoms in both Spiro-OMeTAD and perovskite, giving an atomic level protection in both lateral and vertical direction. The fluorinated PSCs showed water-repellent property, exhibiting the ability to withstand 5 min of water immersion without obvious decomposition. In addition, the PSCs with fluorination treatment exhibited higher stability upon moisture and light exposure. The $\mathrm{CF}_{4}$ plasma has negligible impact on the morphology and electrical properties of Spiro-OMeTAD, resulting in the identical photovoltaic performance as that of the pristine PSC. The fluorinated PSCs without any encapsulation exhibited a PCE of $18.7 \%$ and after continuous working for $100 \mathrm{~h}$ under xenon lamp illumination (contain ultraviolet radiation) in ambient air, the PSCs still retained over $70 \%$ of its initial efficiency, which is $>4000 \%$ improvement compared to conventional PSCs. 
(a)

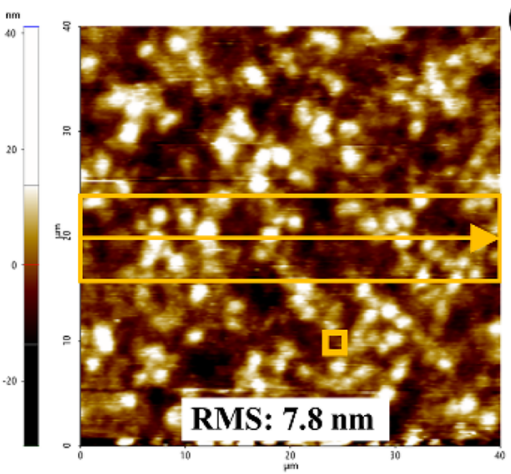

(d)

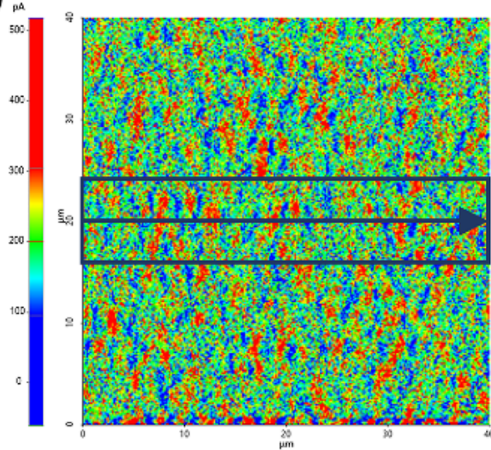

(g)

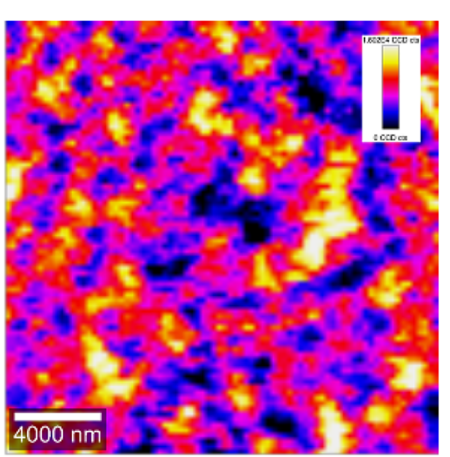

(b)

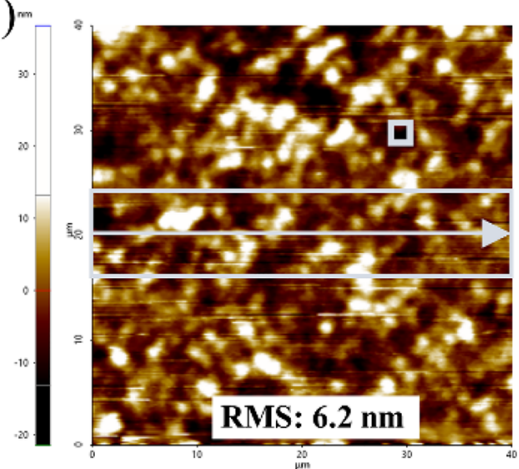

(e)

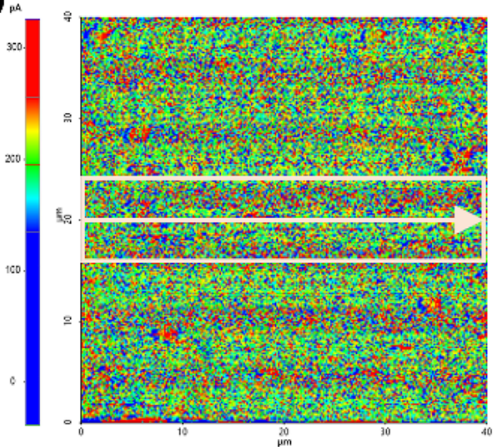

(h)

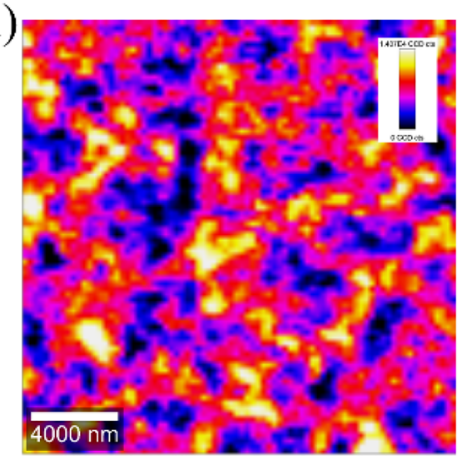

(c)

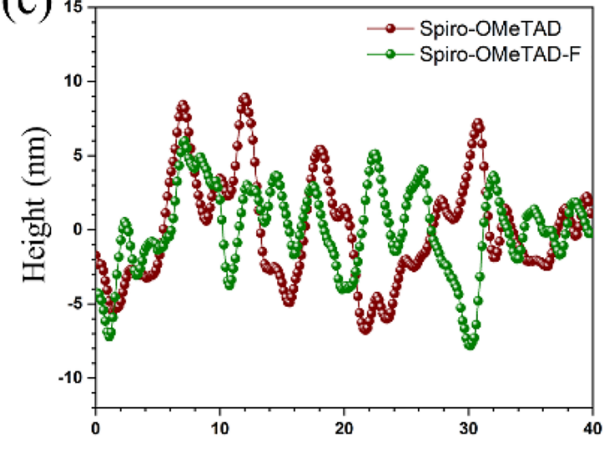

(f)

Distance $(\mu \mathrm{m})$

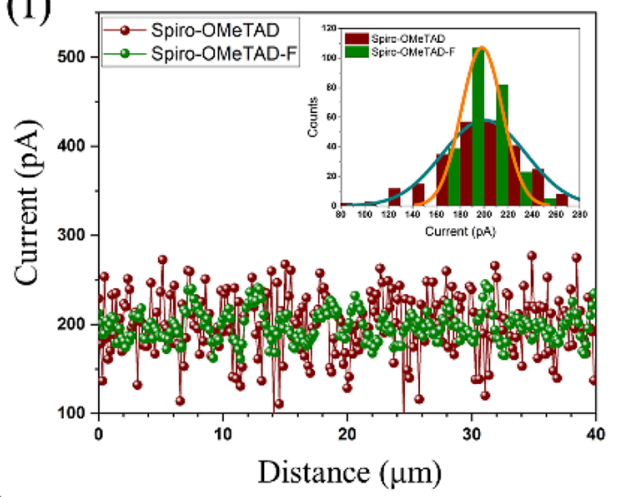

(i)

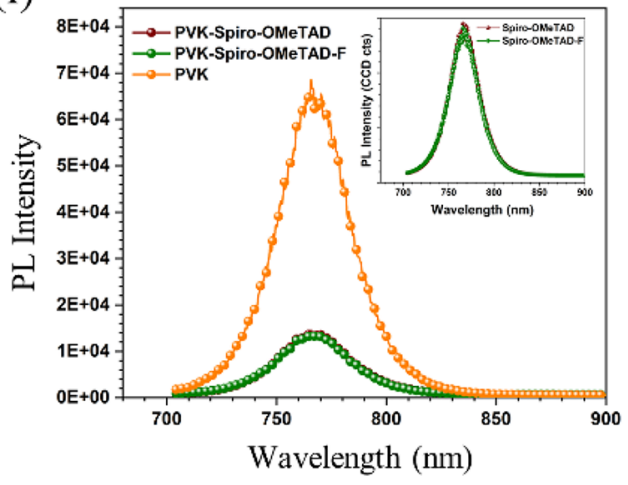

Figure 2. Electronic surface landscape of HTL. AFM height and c-AFM current map of (a,d) Spiro-OMeTAD and (b,e) Spiro-OMeTAD-F thin films coated on $\mathrm{MAPbI}_{3}$ perovskite, (c) height and (f) current profile along the in-plane direction, as specified in the rectangle marked in AFM images, where the inset in (f) shows the statistical distribution of detected current. Confocal photoluminescence map of $(\mathrm{g})$ Spiro-OMeTAD and (h) Spiro-OMeTAD-F coated $\mathrm{MAPbI}_{3}$, depicted with photon energy of $1.6 \mathrm{eV}$. (i) Average steady-state PL spectra of $\mathrm{MAPbI}_{3}$ film, SpiroOMeTAD, and Spiro-OMeTAD-F films coated on perovskite.

Results and Discussion. Plasma fluorination treatment is a facile, controllable, and clean method for the synthesis of fluorinated surfaces with high degree of hydrophobicity. ${ }^{25,26}$ The occurrence process involved in plasma treatment can be determined by the reactivity between the feed gas and substrate material, treatment power, time duration, operating pressure, and so forth. In this work, $\mathrm{CF}_{4}$ plasma was used to fluorinate the perovskite device to achieve an atomic scale coating over all the exterior surfaces. The experimental apparatus of the $\mathrm{CF}_{4}$ fluorination is shown in Figure S1, where the PSC device is placed in the quartz chamber fed with $\mathrm{CF}_{4}$ gas. Upon the plasma ignition assisted by the microwave, radicals, ions, electrons, and other species are generally produced in the plasma. Typically, the free radicals in the plasma are more abundant than the ions because the radicals can be generated at a lower threshold energy and have a longer lifetime. In order to avoid the physical bombardment by the high energetic ions, the treatment power was adjusted as low as $20 \mathrm{~W}$ for achieving the surface fluorination without physical damage. Therefore, in this process, the highly reactive fluorine radicals $\left(\mathrm{F}\right.$ and $\mathrm{CF}_{x}$ ) dominantly react with the active sites of the surface and form stable carbon-fluorine bonds on the exterior surfaces of PSC device. To verify the chemical interaction of $\mathrm{CF}_{4}$ plasma with the top surface of Spiro$\mathrm{OMeTAD}$, X-ray photoelectron spectroscopy (XPS) of the Spiro-OMeTAD films (without LiTFSI and 4-tert-butylpyridine dopant) before and after fluorination was measured to analyze the surface fluorine bonding. Figure 1a shows the XPS survey spectra of the Spiro-OMeTAD films. The pristine SpiroOMeTAD film shows presence of typical $\mathrm{C}, \mathrm{O}$, and $\mathrm{N}$, while the fluorinated Spiro-OMeTAD (Spiro-OMeTAD-F) in addition to $\mathrm{C}, \mathrm{O}$, and $\mathrm{N}$ exhibits a strong $\mathrm{F}$ 1s peak. To quantify the composition and bonding type of the fluorination, high-resolution XPS spectrum of the fluorinated Spiro- 


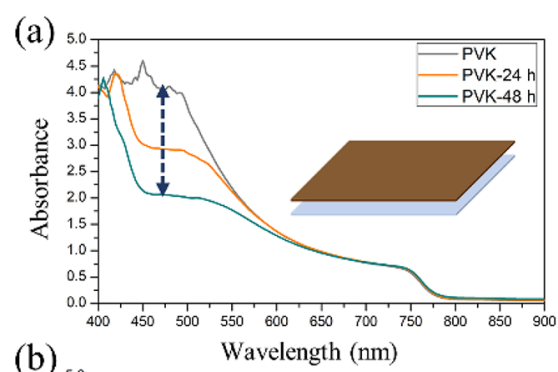

(b)

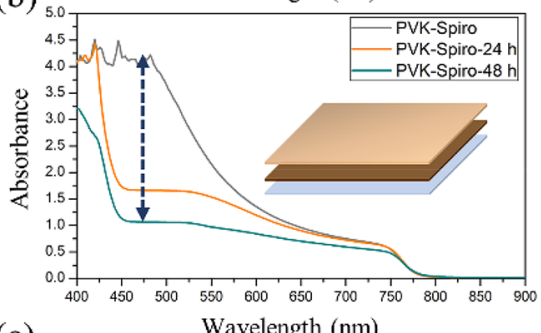

(c)

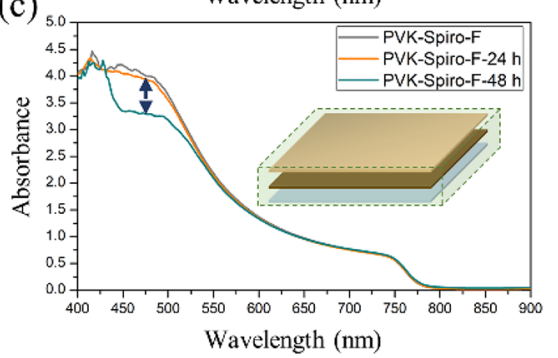

(d)

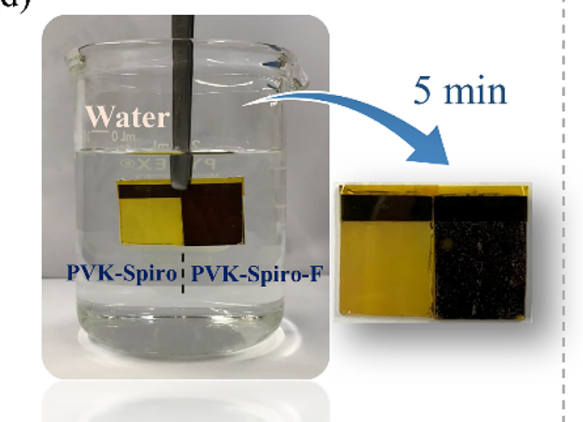

(e)

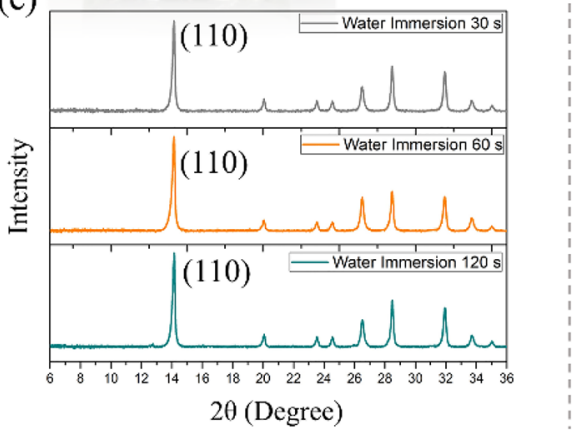

(f)

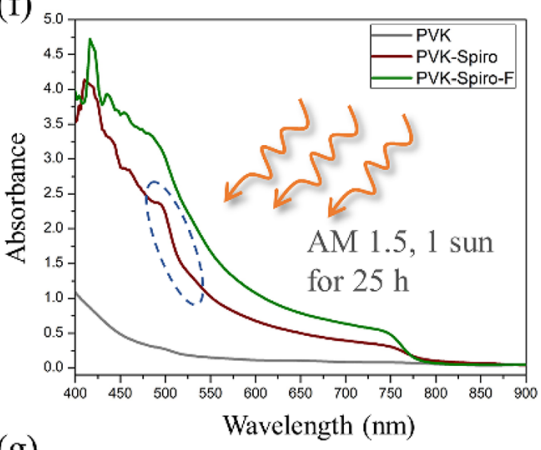

(g)

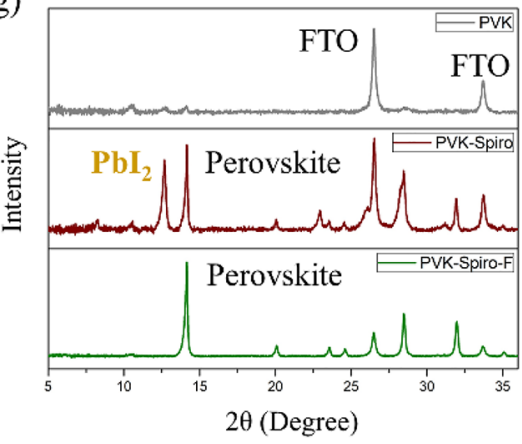

Figure 3. Stability improvement of PSCs by the plasma fluorination treatment. UV-vis absorption spectra of (a) PVK, (b) PVK-Spiro, and (c) PVK-Spiro-F films after $85 \%$ humid air exposure for $48 \mathrm{~h}$. (d) The illustration of 5 min water immersion testing for PVK-Spiro film and PVKSpiro-F film. The photo was taken after water immersion for $2 \mathrm{~min}$. (e) XRD of the fluorinated perovskite/Spiro-OMeTAD films after water immersion for 30, 60, and $120 \mathrm{~s}$. (f) UV-vis absorption spectra and (g) XRD of PVK, PVK-Spiro, PVK-Spiro-F films after AM 1.5G, 1 sun illumination in $36 \%$ humid air for $25 \mathrm{~h}$.

OMeTAD sample was measured. The high-intensity peak for $\mathrm{C}-\mathrm{F}$ bond was observed at $688.0 \mathrm{eV}$ (Figure $1 \mathrm{~b}$ ). In the binding energy region of $280-300 \mathrm{eV}$ (Figure 1c), besides the chemical bonds of $\mathrm{C}-\mathrm{C}(284.5 \mathrm{eV}), \mathrm{C}-\mathrm{N}(285.8 \mathrm{eV}), \mathrm{C}-$ $\mathrm{O}(286.7 \mathrm{eV}), \mathrm{C}=\mathrm{O}(288.0 \mathrm{eV})$, and $\mathrm{COOH}(289.1 \mathrm{eV})$ in Spiro-OMeTAD, the bonds of $\mathrm{CF}_{2}(290.9 \mathrm{eV})$ and $\mathrm{CF}_{3}(292.9$ $\mathrm{eV}$ ) were predominantly observed with an atomic percentage of $38.9 \%$ and $12.8 \%$, respectively. This indicates that the $\mathrm{F}$ atom was easily anchored on the surface of Spiro-OMeTAD by forming $\mathrm{C}-\mathrm{F}_{x}$ bonds during the $\mathrm{CF}_{4}$ plasma treatment. The fluorination of Spiro-OMeTAD can also be verified from the Raman spectra (Figure S2). The band at 1580 and $1600 \mathrm{~cm}^{-1}$ corresponds to the vibration of the aromatic ring system in Spiro-OMeTAD. The ratio of the $1580 \mathrm{~cm}^{-1}$ peak to 1600 $\mathrm{cm}^{-1}$ peak was increased upon the fluorination, suggesting that the fluorine incorporation changed the vibration of the ring chain of Spiro-OMeTAD. ${ }^{26,27}$

The plasma fluorination treatment is capable of providing full protection for the PSCs in all directions. Because all of the exterior surfaces of PSC are exposed to $\mathrm{CF}_{4}$ plasma, as illustrated in Figure 1d, not only the top surface but also the lateral surfaces can be bonded with fluorine. Figure 1e shows the F 1s XPS mapping of the top surface and lateral surface of PSC, where the reddish color dots indicate the area covered with fluorine. It is observed that the top surface of Spiro$\mathrm{OMeTAD}$ was uniformly bonded with the $\mathrm{F}$ atoms, and lateral surface across the layers from Spiro-OMeTAD, perovskite, $\mathrm{TiO}_{2}$ to glass substrate are also bonded with $\mathrm{F}$, which is indicated from the XPS mapping of the lateral surface of PSC.
The fluorine was bonded on $\mathrm{TiO}_{2}$ surface by forming $\mathrm{Ti}-\mathrm{F}$, as indicated in XPS survey and high-resolution spectra of the fluorinated $\mathrm{TiO}_{2}$ layer (Figure S3), where the peak at $684.4 \mathrm{eV}$ was assigned to $\mathrm{Ti}-\mathrm{F}$ bond. ${ }^{28,29}$ In order to verify the composition in different areas in the vertical direction, we selected four individual locations on the lateral surface of PSC from Spiro-OMeTAD to the glass substrate and measured the XPS spectra, as shown in Figure S4. Every location exhibited strong F 1s peak, which suggests that the lateral surfaces across all the layers were covered with fluorine, thereby, providing a full-range of atomic coating on the PSCs. Figure 1f shows the scanning electron microscope (SEM) images of the SpiroOMeTAD surface with and without fluorination. Because the plasma with low ignition power is a mild process, it imparts no damage and these two films exhibited identical surface morphology. Similarly, the thickness of the Spiro-OMeTAD film keeps unchanged after plasma treatment (Figure S5). This is because the plasma treatment only induces the chemical modification on the surface of Spiro-OMeTAD thin film. The absence of noticeable morphology and thickness changes in the film suggests an atomic scale modification rather than a thin film deposition on the surface. Figure S6 shows the UV-vis absorption spectra of the two films, which also showed identical absorption features. To demonstrate the water repellent property, the water contact angle was tested for the Spiro-OMeTAD film with and without fluorination, as shown in Figure 1g. The pristine Spiro-OMeTAD film exhibited a contact angle of $72^{\circ}$, whereas it increased to $116^{\circ}$ after the fluorination treatment, suggesting that the hydrophobicity was 
largely improved upon the fluorine bonding. The results suggest that the atomically bonded fluorine can significantly enhance the water resistance of PSCs and have negligible effect on the morphology and optical properties.

The surface morphology and electrical uniformity of the hole transport layer (HTL) is important for the electrical charge transport throughout the multiple functional layers within the solar cell device. In order to investigate the effect of fluorination on the surface topology and electrical properties of the Spiro-OMeTAD layer, the conductive atomic force microscopy (c-AFM) measurements were performed. Figure $2 \mathrm{a}, \mathrm{b}$ exhibit the topographic images of the Spiro-OMeTAD and Spiro-OMeTAD-F thin films coated on $\mathrm{CH}_{3} \mathrm{NH}_{3} \mathrm{PbI}_{3}$ $\left(\mathrm{MAPbI}_{3}\right)$ perovskite layer. An average height profile was extracted along the in-plane direction from the height map for quantifying the surface homogeneity, as shown in Figure 2c. The Spiro-OMeTAD film displayed a calculated root-mean-square (RMS) of $7.8 \mathrm{~nm}$ while the SpiroOMeTAD-F film exhibited a smaller RMS of $6.2 \mathrm{~nm}$. In comparison to Spiro-OMeTAD, the slight RMS change in the fluorinated Spiro-OMeTAD-F film can be ascribed to the plasma treatment that modified the sample surface by the fluorination reaction. Interestingly, as shown in Figure 2d,e, the Spiro-OMeTAD-F thin film also displays an electrically uniform surface with less current fluctuations, as compared to that of Spiro-OMeTAD. This can be further verified from Figure 2f, where the Spiro-OMeTAD-F displayed fewer deviations in current and a narrower current statistical distribution. Because the $\mathrm{CF}_{4}$ plasma provides an atomic $\mathrm{F}$ layer on top of Spiro-OMeTAD, due to the charge tunneling effect the current collection from HTL will not be affected, so as the overall electrical conductivity of the HTL. This can be verified from the current sweep profile obtained from one spot in HTL (Figure S7).

In addition to film morphology and conductance, the charge extraction from perovskite photoactive layer to HTL is a crucial step for high PCE. To investigate the influence of fluorination on the interfacial charge transfer, we examined the charge extraction at the perovskite/Spiro-OMeTAD interface by measuring the confocal photoluminescence (PL) map of the perovskite/Spiro-OMeTAD films. Figure $2 \mathrm{~g}, \mathrm{~h}$ shows the PL map of the perovskite/Spiro-OMeTAD films with and without fluorination. The average steady PL spectra extracted from the PL map is shown in Figure 2i. The perovskite/SpiroOMeTAD films with and without fluorination treatment showed identical PL spectra with peak intensities drastically smaller than that of non-HTL coated perovskite. These results suggest the fluorination treatment has negligible effects on the charge extraction at the perovskite/Spiro-OMeTAD interface.

The influence of fluorination on the moisture stability of perovskite films $\left(\mathrm{MAPbI}_{3}\right)$ was tested by storing the films in the high humid air with a relative humidity of $85 \%$ and monitoring the UV-vis absorption spectra of the films. We compared three types of films, pristine perovskite film (PVK), perovskite film coated with Spiro-OMeTAD (PVK-Spiro) and perovskite film coated with Spiro-OMeTAD-F (PVK-Spiro-F). The three films exhibited identical absorption initially, as shown in Figure S8. After high humid air exposure for $24 \mathrm{~h}$, due to the formation of perovskite hydrate upon reaction with water ${ }^{30,31}$ the absorbance of perovskite film began to decrease, particularly in the range of $400 \sim 550 \mathrm{~nm}$. In order to compare the absorption behavior among the three films, we selected the absorbance at $475 \mathrm{~nm}$ as the reference. For the PVK film, the absorbance decreased from 4.0 to 3.0 in the first $24 \mathrm{~h}$ and further decreased to 2.0 after $48 \mathrm{~h}$ of humid air exposure, as shown in Figure 3a. The perovskite film coated with SpiroOMeTAD showed inferior stability against moisture. The absorbance at $475 \mathrm{~nm}$ dramatically decreased from 4.0 to 1.7 in the first $24 \mathrm{~h}$ and further to 1.0 in the next $24 \mathrm{~h}$. Moreover, the absorbance in the whole wavelength range decreased noticeably after $48 \mathrm{~h}$ of moisture exposure (Figure $3 \mathrm{~b}$ ). The faster degradation of perovskite with Spiro-OMeTAD is due to the addition of Li-TFSI, which accelerates the water adsorption of the perovskite film. As shown in Figure 3c, fluorination significantly improves the stability of PSCs against humid air. In the first $24 \mathrm{~h}$, the absorbance of PVK-Spiro-F film remained almost identical to the original one, and in the following $24 \mathrm{~h}$ of exposure, the absorption only showed a slight decrease in the wavelength range of $450 \sim 500 \mathrm{~nm}$.

To further demonstrate the water repellent property of the fluorinated PSCs, we conducted accelerated moisture exposure testing by directly immersing the perovskite/Spiro-OMeTAD films into the water and compared the morphological changes as a function of time, as illustrated in Movie S1. The color of pristine perovskite/Spiro-OMeTAD film began to fade after 20 $\mathrm{s}$ in water then gradually converted to yellow and completely turned into yellow $\mathrm{PbI}_{2}$ after $2 \mathrm{~min}$ in water, as shown in Figure $3 \mathrm{~d}$. In contrast, the perovskite/Spiro-OMeTAD-F exhibited a much higher resistance to water, showing no noticeable color change upon immersion. After $5 \mathrm{~min}$ of water immersion, minor spots started to emerge with yellow color while the majority of film remained as the black perovskite. Xray diffraction (XRD) patterns of fluorinated perovskite/SpiroOMeTAD films after water immersion for 30, 60, and $120 \mathrm{~s}$, respectively, are shown in Figure $3 \mathrm{e}$. All the films showed typical perovskite structure peaks without observation of $\mathrm{PbI}_{2}$ peaks, suggesting no perovskite decomposition occurred during the direct contact with water.

In addition to moisture corrosion, light can trigger the perovskite decomposition when exposed to oxygen and moisture. We compared the light stability of PVK, PVKSpiro, and PVK-Spiro-F films by measuring the absorption spectra (Figure 3f) and XRD (Figure 3g) after AM 1.5G, 1 sun illumination in $36 \%$ humid air for $25 \mathrm{~h}$. The PVK film exhibited negligible absorption in the visible light range but showed a cutoff wavelength at around $410 \mathrm{~nm}$. Likewise, in the XRD of PVK film, the perovskite peaks almost disappeared, mainly leaving the FTO diffraction peaks. The absence of the perovskite crystal features is due to the transition from threedimensional (3D) network of octahedra in $\mathrm{MAPbI}_{3}$ crystals to one-dimensional (1D) double-chains perovskite monohydrate $\left(\mathrm{CH}_{3} \mathrm{NH}_{3} \mathrm{PbI}_{3} \cdot \mathrm{H}_{2} \mathrm{O}\right)$ upon the intercalation of water molecules. As evidenced by the enlarged XRD of the PVK film (Figure S9), except for the peaks of the remaining $\mathrm{MAPbI}_{3}$ crystals, the new peak at $10.5^{\circ}$ was observed, which corresponds to the (110) reflections of the monoclinic $\mathrm{CH}_{3} \mathrm{NH}_{3} \mathrm{PbI}_{3} \cdot \mathrm{H}_{2} \mathrm{O}$ hydrate. ${ }^{31,32}$ For the PVK-Spiro films, due to the large amounts of water adsorption by the Li-TFSI dopants in Spiro-OMeTAD, the PVK-Spiro film partially decomposed into $\mathrm{PbI}_{2}$, as indicated from the XRD that peak corresponding to $\mathrm{PbI}_{2}(001)$ plane was observed at $12.7^{\circ}$. The emergence of $\mathrm{PbI}_{2}$ can be confirmed from absorption spectra. The absorption edge around $520 \mathrm{~nm}$ corresponding to $\mathrm{PbI}_{2}$ was detected. In contrast, the light stability of PVK-Spiro-F film was improved as well, which showed identical absorption and crystal structure to that of the original film without 
(a)

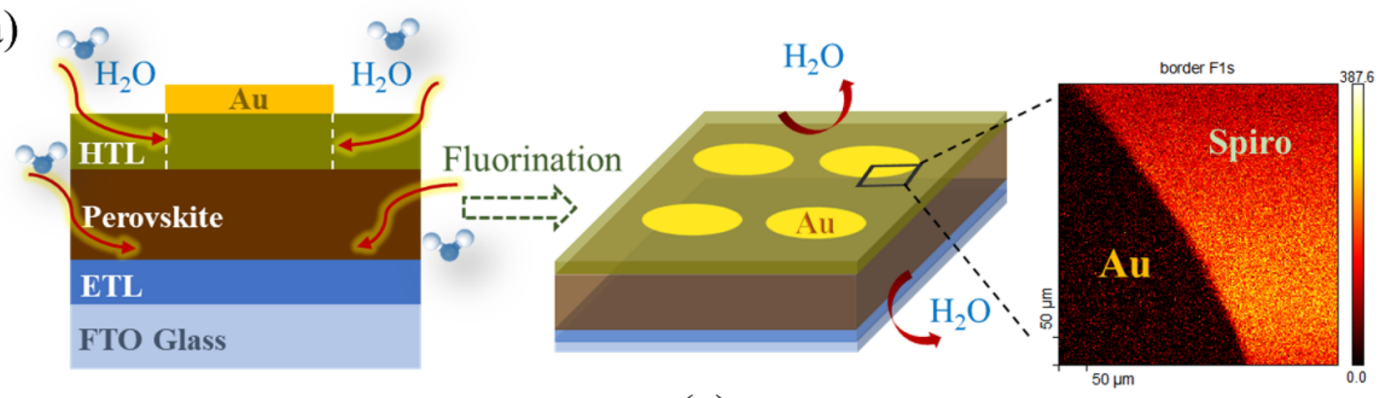

(b)

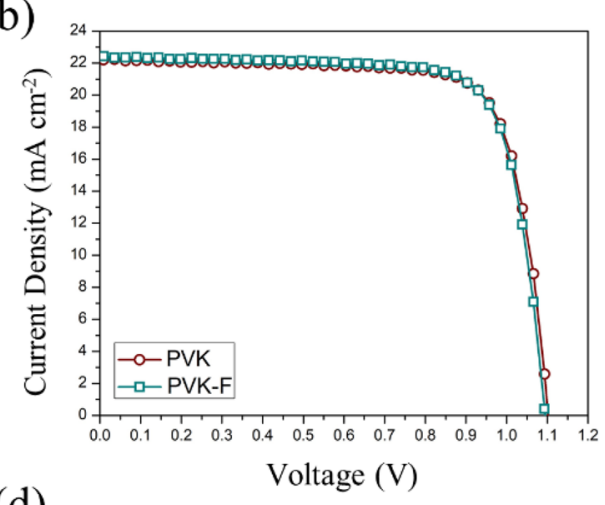

(c)

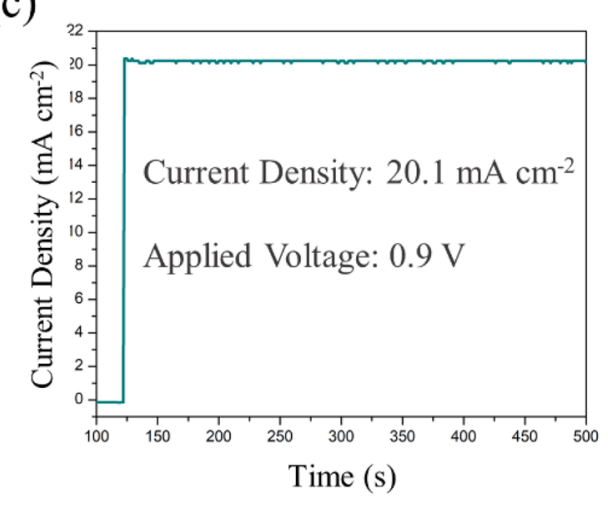

(d)

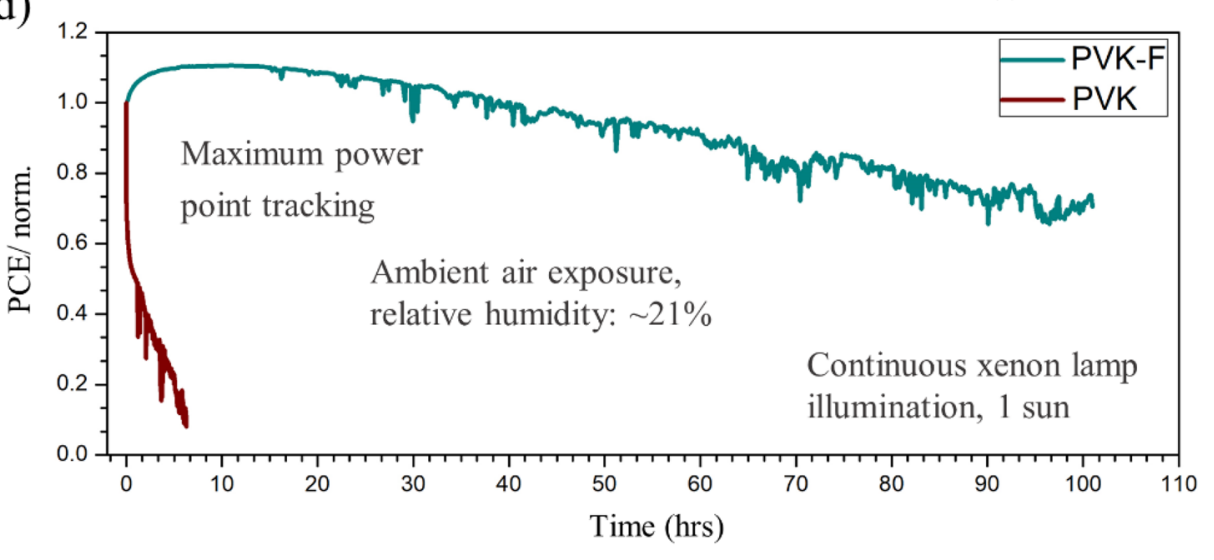

Figure 4. Photovoltaic performance and long-term stability of the PSCs. (a) Schematics of the moisture penetration along the in-plane direction that corrodes the perovskite layer. F 1s XPS mapping of the boundary between Au electrode and Spiro-OMeTAD. (b) $J-V$ characteristics of the PSCs with and without fluorination. (c) The steady conversion efficiency of fluorinated PSC which is measured with a constant potential of 0.9 V. (d) Maximum power point tracking under continuous Xe lamp (AM 1.5G, 1 sun) illumination in ambient air with a relative humidity of $\sim 21 \%$ for $100 \mathrm{~h}$.

decomposition after $25 \mathrm{~h}$ of light soaking, as confirmed by the XRD and UV-vis absorption spectrum.

The results from the high humid air exposure, water immersion, and light soaking tests indicate that the plasma fluorination treatment is an effective method toward improving the stability of PSCs against corrosion by the formation of an impermeable hydrophobic fluorine layer on all the exterior surfaces of PSCs, which provides a full protection.

The stability of PSCs device is not only related to the intrinsic nature of each functional layer but also to the architecture of the PSCs. Metals, such as $\mathrm{Au}, \mathrm{Ag}, \mathrm{Al}$, and so forth, are normally deposited as electrode to collect the charge carriers. The metal electrodes also have a critical effect on the stability of PSCs as they can serve as a barrier layer to block the moisture infiltration into the underneath perovskite. However, in either lab-type cell or module-type cell, there are uncoated areas that are exposed to ambient air, such as the gaps and the edges of the cell, providing penetration path for the water molecules to corrode the perovskite layer, as illustrated in Figure 4a. Additionally, the metal electrode can cause metalcontact-induced degradation since $\mathrm{Au}$ can diffuse across the HTL into the perovskite layer and also halide species can diffuse up to the metal, resulting in irreversible degradation. ${ }^{33-36}$ The plasma fluorination process described here provides solution to address all these problems. As discussed previously, the atomic layer coating of fluorine can be formed on both top and lateral surfaces of PSCs to provide a full protection, which prevents the penetration of water molecules through the gaps, edges, and the interfaces into the perovskite photoactive layer. We measured the F 1s XPS mapping at the boundary between $\mathrm{Au}$ electrode and Spiro-OMeTAD (Figure $4 a)$, where the reddish color indicated fluorine was bonded in that area. It is observed that the boundary between $\mathrm{Au}$ electrode and the Spiro-OMeTAD were fully bonded with fluorine. The corresponding XPS scan shown in Figure S10, demonstrate a strong $\mathrm{F}$ 1s peak in the area without $\mathrm{Au}$. The 
metal electrode coupled with fluorine at the exterior surfaces resulted in the formation of a robust barrier over the whole PSC device. Boyd et al. have reported that volatile halide species in perovskite can escape from the uncovered edges of the active layer into the metal electrode. ${ }^{36}$ In this structure, the whole cell with all the exterior surfaces being coated by the fluorine atoms effectively blocked the interdiffusion.

To examine the photovoltaic performance, we measured the $J-V$ characteristics of the PSCs with and without fluorination, as shown in Figure $4 \mathrm{~b}$. The device showed identical photovoltaic performance before and after fluorination with a $V_{\text {oc }}$ of $1.10 \mathrm{~V}, J_{\mathrm{sc}}$ of $22.1 \mathrm{~mA} \mathrm{~cm} \mathrm{~cm}^{-2}$, and $\mathrm{FF}$ of 0.77 , corresponding to a conversion efficiency of $18.7 \%$. Figure $4 c$ presents the steady current of the fluorinated PSC measured by applying a constant potential of $0.9 \mathrm{~V}$. The steady current density of $20.1 \mathrm{~mA} \mathrm{~cm}{ }^{-2}$ was obtained, corresponding to a steady-state efficiency of $18.1 \%$. The long-term stability was evaluated by tracking the maximum power point of PSCs, as shown in Figure $4 \mathrm{~d}$. In order to mimic the practical operating condition of the solar cells, the unencapsulated PSCs were continuously illuminated by the xenon lamp (AM 1.5G, 1 sun) under ambient air with a relative humidity of $\sim 21 \%$, and the maximum power points of the PSCs were tracked constantly for $100 \mathrm{~h}$. No UV filter was employed, hence the UV radiationinduced potential degradation is also taken into consideration. The degradation behavior of the conventional PSC without fluorination is consistent with the stability test by Domanski et al., that the device showed an initial exponential loss region, followed by a slower linear region. ${ }^{37}$ The initial exponential loss is probably due to the invasion of moisture, forming the perovskite hydrate. The conversion efficiency of the conventional PSC rapidly decreased to $\sim 57 \%$ of initial efficiency in the first $0.3 \mathrm{~h}$ and linearly degraded to $\sim 10 \%$ in the next $6 \mathrm{~h}$ with the linear slope of around $-0.071 \% \mathrm{~h}^{-1}$. In contrast, the PSC with fluorination exhibited a much-improved stability without the rapid exponential degradation, suggesting the initial moisture invasion was effectively prevented by the bonded atomic fluorine layer. The efficiency of the fluorinated PSC showed a linear and slow degradation with a linear slope of around $-0.0045 \% \mathrm{~h}^{-1}$ and after $100 \mathrm{~h}$ of continuous simulated solar illumination, the PSC can still retain over $70 \%$ of its initial efficiency. Taking the elapsed time for obtaining $10 \%$ of its initial efficiency as the comparison standard, the stability for the fluorinated PSCs was improved by more than $4000 \%$. The photovoltaic performance and the long-term stability suggest that the facile plasma fluorination treatment is an effective method toward improving the stability of PSC device without affecting the conversion efficiency.

In conclusion, we report a facile and effective plasma fluorination method to realize a highly durable PSC device. Through the plasma fluorination, the top and lateral surfaces of the PSCs were covered with a strongly bonded $\mathrm{C}-\mathrm{F}_{x}$ layer, providing a robust and full protection. The fluorinated PSC showed excellent stability in various environmental stimuli, such as high humid air, light, and oxygen, and it can even withstand direct contact with water for up to $5 \mathrm{~min}$ without obvious decomposition. The fluorination has a negligible impact on the electrical properties and charge transfer properties of PSCs. Fluorinated PSC exhibited $18.7 \%$ conversion efficiency, which is identical to that of the pristine cell. The atomic fluorine layers over the PSC device efficiently prevented the moisture penetration from both in-plane and out-of-plane directions, significantly improving the stability of
PSCs under operational condition. Under continuous Xe lamp (AM 1.5G, 1 sun) illumination in ambient air for $100 \mathrm{~h}$, the fluorinated PSCs retained $70 \%$ of initial conversion efficiency, which is $4000 \%$ higher than the pristine PSC device.

Methods. Synthesis of $\mathrm{MAPbl}_{3}$ layer. Unlike the conventional perovskite layer that is synthesized from the precursor solution, the $\mathrm{MAPbI}_{3}$ perovskite layer in this work was synthesized through the self-assembly process by slicing and restacking the $\mathrm{MAPbI}_{3}$ crystals. ${ }^{17,38}$ In this process, the $\mathrm{MAPbI}_{3}$ crystals were exfoliated by methylamine $\mathrm{CH}_{3} \mathrm{NH}_{2}$, which was subsequently transformed into liquid two-dimensional layered perovskite intermediate. The perovskite thin film was synthesized by directly spin coating the perovskite intermediate solution. The experimental detail is described as following.

$\mathrm{MAPbI}_{3}$ crystals were placed in a vial which was then sealed into a bottle containing methylamine solution and stored overnight. The black $\mathrm{MAPbI}_{3}$ crystals subsequently turned to a clean yellow liquid perovskite intermediate due to the intercalation of methylamine $\left(\mathrm{CH}_{3} \mathrm{NH}_{2}\right)$ into the $\mathrm{MAPbI}_{3}$ crystals. To dilute the perovskite intermediate, acetonitrile with poly(methyl methacrylate) (PMMA) solution was mixed with the perovskite intermediates to form a $1 \mathrm{M}$ perovskite solution. The perovskite film can be directly obtained by spin coating the solution at $4000 \mathrm{rpm}$ for $30 \mathrm{~s}$, followed by annealing at 100 ${ }^{\circ} \mathrm{C}$ for $10 \mathrm{~min}$. For the synthesis of the conventional $\mathrm{MAPbI}_{3}$ layer, we used the antisolvent method. The $\mathrm{PbI}_{2}$ and MAI (molar ratio 1:1) were dissolved in GBL and DMSO (volume ratio $7: 3$ ) and stirred at $70{ }^{\circ} \mathrm{C}$ to form a $1.2 \mathrm{M}$ solution. After complete dissolution, the solution was filtered and then spin coated at $4000 \mathrm{rpm}$ for $20 \mathrm{~s}$ using toluene as antisolvent, followed by annealing at $100{ }^{\circ} \mathrm{C}$ for $10 \mathrm{~min}$.

PSC Device Fabrication. The compact $\mathrm{TiO}_{2}$ layer was spin coated on cleaned FTO substrate at $2000 \mathrm{rpm}$ for $20 \mathrm{~s}$ using a mildly acidic titanium isopropoxide solution and then heated at $150{ }^{\circ} \mathrm{C}$ for $10 \mathrm{~min}$. Next, the mesoporous $\mathrm{TiO}_{2}$ layer was spin coated on the $\mathrm{TiO}_{2}$ compact layer at $6000 \mathrm{rpm}$ for $30 \mathrm{~s}$ using $\mathrm{TiO}_{2}$ paste which was diluted by $\alpha$-terpineol and ethanol, and then annealed at $500{ }^{\circ} \mathrm{C}$ for $30 \mathrm{~min}$. After deposition of perovskite layer on top of $\mathrm{TiO}_{2}$ electron transport layer, the Spiro-OMeTAD solution was spin coated on the perovskite layer at $4000 \mathrm{rpm}$ for $20 \mathrm{~s}$. Lastly, $80 \mathrm{~nm}$ of gold electrode layer was thermally evaporated on top of Spiro-OMeTAD.

The fluorination was performed by using IoN Wave 10 plasma system (PVA TePla). The PSC device was placed into the quartz chamber of the plasma system and evacuated to a base pressure of $100 \mathrm{mT}$ Trr. Then the $\mathrm{CF}_{4}$ gas was filled into the chamber with a flow rate of 250 SCCM for $1 \mathrm{~min}$ to reach a pressure of $600 \mathrm{mTorr}$. The frequency of the microwave source is $2.45 \mathrm{GHz}$. The ignition power was set up as $20 \mathrm{~W}$ and operated for $30 \mathrm{~s}$ at room temperature to treat the sample.

Device Characterization. X-ray photoelectron spectroscopy (XPS) characterization was performed on a PHI VersaProbe III scanning XPS microscope using monochromatic Al K-alpha $\mathrm{X}$-ray source $(1486.6 \mathrm{eV})$. XPS measurements of lateral surface of PSC (Figure S4) were acquired using $10 \mu \mathrm{m} / 100 \mathrm{~W} / 15 \mathrm{kV}$ $\mathrm{X}$-ray setting and dual-beam neutralization. In order to exclude the presence of fluorine in the FTO glass, blank glass slides were used for the XPS characterization. All other spectra were acquired with $10 \mu \mathrm{m} / 100 \mathrm{~W} / 20 \mathrm{kV} \mathrm{X}$-ray setting and dualbeam neutralization over $1400 \mu \mathrm{m} \times 100 \mu \mathrm{m}$ sample area. Pass energy of $26 \mathrm{eV}$ was used in all measurements, which gives a $\mathrm{Ag} 3 \mathrm{~d}_{5 / 2}$ full width at half-maximum of $0.59 \mathrm{eV}$. All binding 
energies were referenced to $\mathrm{C}-\mathrm{C}$ peak at $284.5 \mathrm{eV}$. XPS mapping was conducted over $200 \mu \mathrm{m} \times 200 \mu \mathrm{m}$ to $1000 \mu \mathrm{m} \times$ $1000 \mu \mathrm{m}$ area with $10 \mu \mathrm{m} / 1.25 \mathrm{~W} / 15 \mathrm{kV}$ X-ray beam. Conductive atomic force microscopy (c-AFM) images were measured by Park XE7 Atomic Force Microscope with the external I-AFM model. Measurements were carried out at a bias voltage of $2 \mathrm{~V}$ and scan rate of $0.5 \mathrm{~Hz}$. X-ray diffraction (XRD) analyses were conducted using Philips Xpert Pro X-ray diffractometer (Almelo, The Netherlands). UV-vis absorption spectra were recorded using U-4100, Hitachi UV-vis spectrophotometer. Steady-state photoluminescence (PL) and mapping was conducted using WITec $300 \mathrm{R}$ system with $633 \mathrm{~nm}$ excitation. Scanning electron microscopy (SEM) images were obtained using scanning electron microscopy (SEM, Quanta 600 FEG, FEI). $J-V$ characteristics of the solar cells were recorded by a Keithley digital source meter (Model 2400) under 1 sun (AM $1.5 \mathrm{G}, 100 \mathrm{~mW} / \mathrm{cm}^{2}$ ) illumination provided by a solar simulator (150 W Sol 2ATM, Oriel).

\section{ASSOCIATED CONTENT}

\section{S Supporting Information}

The Supporting Information is available free of charge on the ACS Publications website at DOI: 10.1021/acs.nanolett.8b04778.

Schematics of the plasma fluorination apparatus; Raman spectra of the Spiro-OMeTAD layers with and without fluorination; XPS spectra of four different locations on the lateral surface of PSC device; cross-sectional SEM of the spiro-OMeTAD layer deposited on FTO glass without and with plasma fluorination treatment; UVvis absorption spectra and current sweep profile of the Spiro-OMeTAD layers with and without fluorination; UV-vis absorption spectra of perovskite layers with and without spiro-OMeTAD layer; enlarged XRD of the PVK film from $5^{\circ}$ to $20^{\circ}$ after 1 sun illumination in $36 \%$ humid air for $25 \mathrm{~h}$; XPS spectra at the Au electrode and the boundary between Au and Spiro-OMeTAD (PDF) Immersion of perovskite/Spiro-OMeTAD films into water, comparing morphological changes as a function of time (AVI)

\section{AUTHOR INFORMATION}

\section{Corresponding Authors}

*E-mail: cuw635@psu.edu.

*E-mail: sup103@psu.edu.

\section{ORCID}

Congcong Wu: 0000-0003-0771-2898

Xu Feng: 0000-0003-1945-1605

Dong Yang: 0000-0002-0518-019X

\section{Author Contributions}

C.W. and K. W. contributed equally to this work.

\section{Notes}

The authors declare no competing financial interest.

\section{ACKNOWLEDGMENTS}

C.W. acknowledges the financial support from the SBIR program through Nanosonic inc. S.P. would like to acknowledge the financial support from Office of Naval Research (I. Perez) through grant number N000141613043. Y.J. was supported through the AMRDEC participation in NSF I/ UCRC: Center for Energy Harvesting Materials and Systems
(CEHMS). Y.Y. would like to acknowledge the support from DARPA MATRIX program. K.W. and Y.H. acknowledge the financial support through Air Force Office of Scientific Research Natural Materials and Biophysics programs under award number FA9550-17-1-0341. XPS analysis was supported by the National Science Foundation under Grant CHE1531834 .

\section{REFERENCES}

(1) Kojima, A.; Teshima, K.; Shirai, Y.; Miyasaka, T. J. Am. Chem. Soc. 2009, 131, 6050-6051.

(2) NREL, Research Cell Record Efficiency Chart, https://www. nrel.gov/pv/assets/pdfs/pv-efficiency-chart.20190103.pdf, (accessed: January 2019).

(3) Zhu, Z.; Hadjiev, V. G.; Rong, Y.; Guo, R.; Cao, B.; Tang, Z.; Qin, F.; Li, Y.; Wang, Y.; Hao, F.; Venkatesan, S.; Li, W.; Baldelli, S.; Guloy, A. M.; Fang, H.; Hu, Y.; Yao, Y.; Wang, Z.; Bao. Chem. Mater. 2016, 28, 7385-7393.

(4) Bryant, D.; Aristidou, N.; Pont, S.; Sanchez-Molina, I.; Chotchunangatchaval, T.; Wheeler, S.; Durrant, J. R.; Haque, S. A. Energy Environ. Sci. 2016, 9, 1655-1660.

(5) Aristidou, N.; Eames, C.; Sanchez-Molina, I.; Bu, X.; Kosco, J.; Islam, M. S.; Haque, S. A. Nat. Commun. 2017, 8, 15218.

(6) Kim, N.-K.; Min, Y. H.; Noh, S.; Cho, E.; Jeong, G.; Joo, M.; Ahn, S.-W.; Lee, J. S.; Kim, S.; Ihm, K.; Ahn, H.; Kang, Y.; Lee, H.-S.; Kim, D. Sci. Rep. 2017, 7, 4645.

(7) Weerasinghe, H. C.; Dkhissi, Y.; Scully, A. D.; Caruso, R. A.; Cheng, Y. B. Nano Energy 2015, 18, 118-125.

(8) Matteocci, F.; Cinà, L.; Lamanna, E.; Cacovich, S.; Divitini, G.; Midgley, P. A.; Ducati, C.; Di Carlo, A. Nano Energy 2016, 30, 162172.

(9) Hwang, I.; Jeong, I.; Lee, J.; Ko, M. J.; Yong, K. ACS Appl. Mater. Interfaces 2015, 7, 17330-17336.

(10) Li, X.; Dar, M. I.; Yi, C.; Luo, J.; Tschumi, M.; Zakeeruddin, S. M.; Nazeeruddin, M. K.; Han, H.; Grätzel, M. Nat. Chem. 2015, 7, 703-711.

(11) Bi, D.; Gao, P.; Scopelliti, R.; Oveisi, E.; Grätzel, M.; Hagfeldt, A.; Nazeeruddin, M. K. Adv. Mater. 2016, 28, 2910-2915.

(12) Hsu, H.-L.; Chang, C.-C.; Chen, C.-P.; Jiang, B.-H.; Jeng, R.-J.; Cheng, C.-H. J. Mater. Chem. A 2015, 3, 9271-9277.

(13) Christians, J. A.; Schulz, P.; Tinkham, J. S.; Schloemer, T. H.; Harvey, S. P.; Tremolet de Villers, B. J.; Sellinger, A.; Berry, J. J.; Luther, J. M. Nat. Energy 2018, 3, 68-74.

(14) Grancini, G.; Roldán-Carmona, C.; Zimmermann, I.; Mosconi, E.; Lee, X.; Martineau, D.; Narbey, S.; Oswald, F.; De Angelis, F.; Graetzel, M.; Nazeeruddin, M. K. Nat. Commun. 2017, 8, 15684.

(15) Kim, Y. C.; Yang, T.-Y.; Jeon, N. J.; Im, J.; Jang, S.; Shin, T. J.; Shin, H.-W.; Kim, S.; Lee, E.; Kim, S.; Noh, J. H.; Seok, S. I.; Seo, J. Energy Environ. Sci. 2017, 10, 2109-2116.

(16) Wang, Z.; McMeekin, D. P.; Sakai, N.; Reenen, S. V.; Wojciechowski, K.; Patel, J. B.; Johnston, M. B.; Snaith, H. J. Adv. Mater. 2017, 29, 1604186.

(17) Wu, C.; Li, H.; Yan, Y.; Chi, B.; Felice, K. M.; Moore, R. B.; Magill, B. A.; Mudiyanselage, R. R. H. H.; Khodaparast, G. A.; Sanghadasa, M.; Priya, S. Sol. RRL 2018, 2, 1800052.

(18) Lee, I.; Yun, J. H.; Son, H. J.; Kim, T.-S. ACS Appl. Mater. Interfaces 2017, 9, 7029-7035.

(19) Pellaroque, A.; Noel, N. K.; Habisreutinger, S. N.; Zhang, Y.; Barlow, S.; Marder, S. R.; Snaith, H. J. ACS Energy Lett. 2017, 2, 2044-2050.

(20) Liu, J.; Wu, Y.; Qin, C.; Yang, X.; Yasuda, T.; Islam, A.; Zhang, K.; Peng, W.; Chen, W.; Han, L. Energy Environ. Sci. 2014, 7, 29632967.

(21) Bella, F.; Griffini, G.; Correa-Baena, J.-P.; Saracco, G.; Gratzel, M.; Hagfeldt, A.; Turri, S.; Gerbaldi, C. Science 2016, 354, 203-206.

(22) Kim, G. H.; Jang, H.; Yoon, Y. J.; Jeong, J.; Park, S. Y.; Walker, B.; Jeon, I. Y.; Jo, Y.; Yoon, H.; Kim, M.; Baek, J.-B.; Kim, D. S.; Kim, J. Y. Nano Lett. 2017, 17, 6385-6390. 
(23) Luo, J.; Xia, J.; Yang, H.; Chen, L.; Wan, Z.; Han, F.; Malik, H. A.; Zhu, X.; Jia, C. Energy Environ. Sci. 2018, 11, 2035-2045.

(24) Wang, Q.; Chen, B.; Liu, Y.; Deng, Y.; Bai, Y.; Dong, Q.; Huang, J. Energy Environ. Sci. 2017, 10, 516-522.

(25) Woodward, I.; Schofield, W. C. E.; Roucoules, V.; Badyal, J. P. S. Langmuir 2003, 19, 3432-3438.

(26) Chaiwong, C.; Rachtanapun, P.; Wongchaiya, P.; Auras, R.; Boonyawan, D. Surf. Coat. Technol. 2010, 204, 2933-2939.

(27) Ferrari, A. C.; Rodil, S. E.; Robertson, J. Phys. Rev. B: Condens. Matter Mater. Phys. 2003, 67, 155306.

(28) Sultana, T.; Georgiev, G. L.; Auner, G.; Newaz, G.; Herfurth, H. J.; Patwa, R. Appl. Surf. Sci. 2008, 255, 2569-2573.

(29) Pan, J. H.; Zhang, X.; Du, A. J.; Sun, D. D.; Leckie, J. O. J. Am. Chem. Soc. 2008, 130, 11256-11257.

(30) Song, Z.; Abate, A.; Watthage, S. C.; Liyanage, G. K.; Phillips, A. B.; Steiner, U.; Grätzel, M.; Heben, M. J. Adv. Energy Mater. 2016, $6,1600846$.

(31) Leguy, A. M. A.; Hu, Y.; Campoy-Quiles, M.; Alonso, M. I.; Weber, O. J.; Azarhoosh, P.; van Schilfgaarde, M.; Weller, M. T.; Bein, T.; Nelson, J.; Docampo, P.; Barnes, P. R. F. Chem. Mater. 2015, 27, 3397-3407.

(32) Manser, J. S.; Saidaminov, M. I.; Christians, J. A.; Bakr, O. M.; Kamat, P. V. Making and Breaking of Lead Halide Perovskites. Acc. Chem. Res. 2016, 49, 330-338.

(33) Guerrero, A.; You, J.; Aranda, C.; Kang, Y. S.; Garcia-Belmonte, G.; Zhou, H.; Bisquert, J.; Yang, Y. ACS Nano 2016, 10, 218-224.

(34) Sanehira, E. M.; Tremolet De Villers, B. J.; Schulz, P.; Reese, M. O.; Ferrere, S.; Zhu, K.; Lin, L. Y.; Berry, J. J.; Luther, J. M. ACS Energy Lett. 2016, 1, 38-45.

(35) Domanski, K.; Correa-Baena, J. P.; Mine, N.; Nazeeruddin, M. K.; Abate, A.; Saliba, M.; Tress, W.; Hagfeldt, A.; Grätzel, M. ACS Nano 2016, 10, 6306-6314.

(36) Boyd, C. C.; Cheacharoen, R.; Bush, K. A.; Prasanna, R.; Leijtens, T.; McGehee, M. D. ACS Energy Lett. 2018, 3, 1772-1778.

(37) Domanski, K.; Alharbi, E. A.; Hagfeldt, A.; Grätzel, M.; Tress, W. Nat. Energy 2018, 3, 61-67.

(38) Wu, C.; Li, H.; Yan, Y.; Chi, B.; Pu, J.; Li, J.; Sanghadasa, M.; Priya, S. Nano Energy 2017, 36, 295-302. 